\title{
Risk sensitivity and assortment in social dilemmas
}

\author{
Michael Kirley • Friedrich Burkhard von der Osten
}

the date of receipt and acceptance should be inserted later

\begin{abstract}
Uncertainty and risk are key features of many social dilemmas, where individual decisions are often made with imperfect knowledge and variance in outcomes. In this paper, we investigate the evolutionary dynamics of the well-known public goods game using a recently introduced modelling framework encapsulating risk sensitive assortment. Here, the population of mobile agents playing the game is divided into fixed-sized interaction groups. Individuals are defined by a single genetic trait - a risk sensitivity trait - that guides their decision-making. This trait is mapped to a continuous range of investment levels and also provides a mechanism to guide mobility (migration) decisions. Detailed computational simulation experiments confirm the relationship between risk orientation, decision-making and mobility in the game. As the size of each group increases, assortment levels tend to decrease and riskaverse individuals tend to dominate the population. However, in many scenarios, there was high variance in the proportion of 'cooperators' both in groups and between different groups suggesting that risk-seeking bevaviour is an emergent property of mobility induced positive assortment.
\end{abstract}

This is a revised and extended version of the SEAL 2014 conference paper by Kirley and von der Osten (2014).

\footnotetext{
M. Kirley

Department of Computing and Information Systems

The University of Melbourne

E-mail: mkirley@unimelb.edu.au

F.B. von der Osten

Department of Computing and Information Systems

The University of Melbourne

E-mail: fvon@unimelb.edu.au
}

\section{Introduction}

Social dilemmas, that is situations in which collective and private interest conflict, have been studied extensively in many domains, including evolutionary biology, social psychology, economics, statistical physics, and multi-agent systems (Hauert, 2006; Nowak, 2012; Perc et al., 2013; Fu et al., 2007; Szolnoki, 2009; Smaldino and Lubell, 2011; Capraro, 2013). The public goods game (Olson, 1971; Gintis, 2000) is a well-known abstract mathematical framework used to investigate mechanisms promoting cooperative behaviour in social dilemmas. In this game, individual participants have to make an investment decision, that is, they must choose how much of their endowment they wish to contribute to the group pool and how much they wish to keep for themselves. The sum of the contributions is multiplied by a constant factor (greater than 1), before then being shared out equally between all group members regardless of their relative contributions. The social dilemma arises as this multiplication factor is usually less than the group size. Thus, there is a temptation for individual agents to 'free-ride,' and the population may evolve toward the 'tragedy of the commons' (Hardin, 1968).

The literature on multi-player social dilemma games is quite diverse. However, the overarching goal of such studies has been to investigate the promotion and stabilisation of cooperation in the population, typically by categorising individuals into 'pro-self' or 'prosocial' types, or by proposing alternative decisionmaking strategies based on reputation and/or interaction topologies. In the case where individuals are faced with a binary decision, either to make a contribution or to refuse to make a contribution, groups of cooperators (or investors) outperform groups of non-cooperators, 
but selfish individuals always do better than cooperators in their group (Nowak, 2012; Perc et al., 2013).

A number of hypotheses explaining why individuals might contribute to a public good, including reciprocal altruism, reputation, punishment, spatial selection and multi-level selection have been proposed (Nowak, 2006, 2012). Additionally, a number of studies have examined the effects of 'mobility patterns' using network or spatial models (Traulsen and Nowak, 2006; Boyd and Richerson, 2002). The rationale behind such models is primarily based on the mechanism underlying positive assortment, where altruistic individuals are likely to help other altruists within their group. In many circumstances, between-group variation exceeds within-group variation, which in turn influences the evolutionary trajectory of the population (Wilson and Dugatkin, 1997). Group success encourages the common belief that everything is going well, consequently no modification of behaviour is required (Macy, 1995). However, individuals not satisfied with their current group may attempt to swap groups in search of 'greener pastures' (Le Galliard et al., 2005; Killingback et al., 2006).

One important direction that requires further research is the role of 'risk' in decision-making, and subsequently the population dynamics and emergent behaviour in social dilemmas. Uncertainty and risk, including risk aversion, risk seeking and risk preference, are key features of many social dilemmas (Kanagaretnam et al., 2009; Parks, 2004; Van Assen and Snijders, 2004). Recently, agent-based models investigating the evolution of risk taking (Stern, 2010) and risk aversion (Hintze et al., 2015) have appeared. Starting from an assumption that risk-seeking behaviour is not favoured by natural selection, inherent biases guiding decisionsmaking strategies have been examined. Hintze et al. (2015) report that beneficial risk aversion adaptations emerge in small populations, particularly when a larger population is divided into smaller groups, with limited migration between groups.

In this paper, we combine the risk sensitivity model introduce by Hintze et al. (2015) with the multiple group public goods game framework used by both Killingback et al. (2006) and Janssen and Goldstone (2006), to investigate evolutionary dynamics. In our simple model, the population of mobile agents is divided into fixed-sized interaction groups. Individuals have a single genetic trait - a risk sensitivity trait - that guides their decision-making. Instead of using a discrete 'cooperate' or 'defect' investment option, the risk sensitivity trait guides a continuous investment strategy. Individuals with a high trait value are considered to be risk averse, corresponding to a low chance of investing a small amount. In contrast, individuals with a low trait value are considered to have a risk seeking strategy, corresponding to a high chance of investing a large amount. At the end of each game playing round, an opportunity arises for individuals to modify their risk sensitivity trait. An individual can update their risk trait by imitating successful (fitter) individuals within their current group. Thus, an individual's risk trait is inherited over generations and is subject to mutation. Individuals are also presented with an opportunity to switch groups at the end of each round of the game. We examine different mobility or switching mechanisms: random migration, conditional migration and risk sensitive migration.

We use Monte Carlo simulation experiments to investigate model behaviour, as it is not practical to study this group-structured model analytically. Key model parameters include population size, the number of groups, and migration/switching mode. The use of a real-value risk sensitivity trait generates rich dynamics. We report results illustrating the evolutionary trajectory of the risk trait, and indirectly the average investment level. Across the population as a whole, the average value of the risk aversion trait is significantly lower in smaller groups and in the random migration model. We find that lower levels of investment are typically maintained in the conditional migration model suggesting that individuals who make decisions based on whether that are satisfied with their local neighbourhood tend to be more risk averse. Detailed analysis of variation of the distribution of the risk aversion trait, measured in terms of the assortment ratio, indicates that positive assortment is more clearly defined in smaller populations.

The remainder of this paper is organised as follows. In Section 2, we briefly discuss background literature describing risk in social dilemmas. Related work describing mobility mechanisms in social dilemmas is also discussed. Our hybrid model is introduced in Section 3. In Section 4, the simulation experiments are described and results presented. We summarise the results and discuss the implications of our findings, before briefly outlining avenues for future work in Section 5 .

\section{Background and related work}

\subsection{Decision-making and risk in social dilemmas}

In social dilemmas, there is an inherent risk in each decision that is made. The cooperative choice can be conceived as a 'gamble' and defection as a 'safe' choice, framed with respect to different reference points. For example, in the public goods game the temptation to 'free-ride' on the contributions of the other players is often seen as the less risky option, consistent with Nash 
equilibrium of the game. Making an investment (cooperating) contains an element of risk no matter which reference point one chooses.

A number of studies focussed on the well-known prisoners' dilemma game have examined risk sensitivity and the evolution of cooperation. For example, Parks (2004) suggested that cooperation in the non-iterative prisoners' dilemma game is a 'risky' behaviour. It was reported that risk-seeking individuals were more cooperative than risk-averse individuals in a five-person prisoner's dilemma game when the risk of others being cooperative was high. However, in the iterative game, Van Assen and Snijders (2004), showed that risk-averse people tended to cooperate when group membership did not change throughout over the course of the game. In a recent study Zeng et al. (2014), incorporate risk attitudes into an iterated prisoner's dilemma game. Here, risk was defined as the standard deviation of the onemove payoffs for players. Interestingly, their simulation results show that risk-averse players perform better than risk-seeking players, a results consists with the work of (Van Assen and Snijders, 2004). Despite the fact that differences in emergent outcomes between repeated games and 'single-shot' games exists, the work of Capraro et al. (2014) highlights the effectiveness of heuristics when used to guide decision-making give variations in possible outcomes.

More generally, the 'risk orientation' of an individual may be defined as an individual's general preference towards making decisions in uncertain situations. Genetic factors have been used to help explain this observation in human experiments, together with environmental variables and individual behavioural histories (Bateson, 2002; Bell, 2009). From a social psychological science perspective, people tend to be risk-averse when dealing with outcomes that are gains relative to their reference point - they choose sure smaller gains over larger riskier gains, but became risk-seeking when dealing with losses (Kahneman and Tversky, 1979). This is in contrast to the widely accepted economic perspective, where a rational decision-maker is an individual who attempts to maximise their expected utility in any decision-making scenario.

\subsection{Mobility in social dilemmas}

It is now widely accepted that mobility (migration) plays an important role in evolutionary dynamics in group structured social dilemmas (Fu and Nowak, 2013; Nowak, 2012). Mobility mechanisms are typically subject to stringent rules, taking into account personal preferences of individuals, their strategies, as well as their aims (Vainstein et al., 2007). In a recent paper,
Gavrilets (2015), have identified that both the composition and size of the group as well as the mechanism used to transfer 'effort' into 'rewards' contribute to the likelihood of the group's success and the level of withingroup 'free riding' in collective social dilemmas. Wilson and Dugatkin (1997) suggest that population viscosity and the effects of group membership and success, plays a significant role in a 'multi-level' selection framework. In addition, Hauert et al. (2014) shows that rare migration can in fact favour cooperation. Under positive assortment, altruistic individuals are likely to help other altruists (Traulsen and Nowak, 2006; Boyd and Richerson, 2002), thus promoting higher levels of cooperation across the population as a whole. In contrast, Traulsen and Nowak (2006) show that migration may have a negative effect on the trajectory of evolving population as it brings about the mixing of groups.

There are still many unanswered questions about the effects of different modes of migration of individuals between groups (Pichugin et al., 2015). In a random mobility model, there is a periodic exchange of individuals between specific groups. This approach is somewhat limited, thus a number of extensions to this base-line model have been proposed and evaluated. Notable examples include success-driven migration (Helbing and $\mathrm{Yu}, 2009$ ), adaptive migration (Jiang et al., 2010) and aspiration-induced migration (Yang et al., 2010). Success-driven migration is based on the idea that individuals can elect to move to sites with higher expected payoffs. The challenge of success-driven migration, however, is to determine in advance the potential payoff of the 'non-local' site. In contrast, in the adaptive migration model individuals only make use of local information when attempting a move. In the study presented by Jiang et al. (2010), adaptive migration took place probabilistically in proportion to the number of defectors in the neighbourhood. Aspiration-induced migration is a third alternative, where individuals move to a new site if their payoff is below a certain aspiration level. Each of these migration schemes has been shown to enhance the extent of cooperative behaviour considerably, even in a noisy environment (Helbing and $\mathrm{Yu}, 2009)$ or in an environment dominated by defectors (Jiang et al., 2010).

In the context of multi-player social dilemma games, the efficacy of conditional mobility mechanisms has been reported in recent work by Chiong and Kirley $(2012,2013)$. However, that work was restricted to spatial models (regular lattice). Works investigating evolutionary dynamics in populations divided into subgroups focussed on the public goods game include the work of Killingback et al. (2006); Janssen and Goldstone (2006). These studies report on the effects of variable groups 
sizes and the impacts of random migration mechanisms on the emergent cooperation levels.

In another interesting related study, Le Galliard et al. (2005) modelled the simultaneous evolution of mobility and altruism. When allowed to evolve separately, altruism was associated with low mobility. Here, constraints on mobility coupled with the life history of genetic traits directly impacted in the coevolution of altruism and mobility.

\section{Model}

\subsection{Overview}

Our model is an evolutionary game theoretic model, which combines the multiple group public goods game investigated by Killingback et al. (2006) and Janssen and Goldstone (2006) and the risk sensitivity model introduce by Hintze et al. (2015). The model consists of a population of agents playing a multiple group version of the continuous public goods game. The population is composed of $g$ disjoint fixed-sized interaction groups, each of size $n$. In each round of the game, each individual must make two separate decisions. Firstly, they must decide how much to invest into the common public pool. Then, at the end of the round, they must decide whether to switch groups or stay in their current group. Each of these decisions is inherently risky. Fig. 1 provides a schematic overview of the multiple group public goods game.

It is reasonable to expect that the fixed-sized trait groups in our model should (a) generated evolutionary trajectories similar to the risk sensitivity model of Hintze et al. (2015), and (b) the emergent 'cooperation levels' will be different to the variable-sized groups used in the models by Killingback et al. (2006).

\subsection{Public goods game with continuous strategy space}

Typically, studies of social dilemmas assume that individuals playing the game have a discrete choice, either to 'cooperate' or to 'defect.' However, this binary decision is somewhat unrealistic, especially when investment levels are considered in games such as the public goods game. We suggest that individual game playing agents are diverse and subsequently can make different decisions when confronted with variance in outcomes. Therefore, we extend the actions available to an individual by taking into account the whole continuous range of the strategy space (Killingback et al., 2006; Janssen and Goldstone, 2006; Hauert, 2006). At an abstract level, this approach has some similarities with

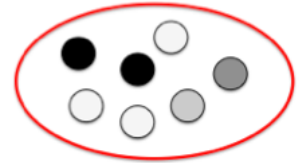

(a)

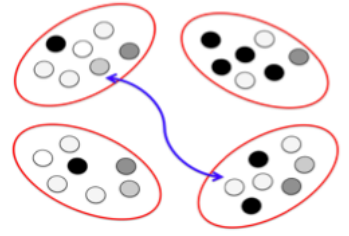

(b)
Fig. 1: Schematic overview of the multiple-group public goods game. (a) A single group public goods game $(g=1)$ with seven players $(n=7)$. The grey-scale circles represent the risk sensitivity trait $(\chi)$ and thus investment level $(x)$ as described in Section 3.2. (b) A multiple-group public goods game with four groups $(g=4)$, each with seven players $(n=7)$. Here, the blue arrow indicates two individuals from separate groups that wish to switch groups. Section 3.5 describes alternative migration or 'group switching' mechanisms.

the probabilistic participation framework introduced in (Sasaki et al., 2007).

Assume that $n$ individuals each make an investment $x_{i}$ in a public good, where each $x_{i} \in(i=1, \ldots, n)$ is a real number between 0 and some positive maximum value $V$. The payoff to individual $i$ is given by:

$\pi_{i}=(k / n) \sum_{j=1}^{n} x_{j}-x_{i}$

where $k$ is a positive constant (which can be viewed as a synergy factor or interest rate). When $1<k<n$ every individual will maximise their payoff by making a zero investment, irrespective of the investments made by the other individuals (that is, act as a free-rider). If all the players make a zero investment, they each receive a payoff 0 . If all the players invest $V$, each would receive $(k-1) \times V$. Here, the ratio $k / n$ is frequently referred to as the 'marginal per capita return' (MPCR) (Shank et al., 2015).

The evolutionary dynamics of the game can be described by the adaptive replicator dynamics for a continuous strategy space (Doebeli et al., 2004; Cressman et al., 2012). Under such conditions, the replicator equation assumes that the population state is described by a Borel probability measure $\mathrm{P}$ over $[0, V]$. The expected payoff of an individual playing $x_{i}$ in a group on $n$ players, where the other $n-1$ players are chosen at random is:

$\pi\left(x_{i}, P\right)=V+\left(\frac{k}{n}-1\right) x_{i}+\frac{k}{n} \bar{x}(n-1)$ 
where $\bar{x}=\int_{[0, V]} x_{j} P\left(d x_{j}\right)$ is the average contribution of an individual in the population.

\subsection{Risk sensitivity trait: decision-making}

Each agent is defined by real-value genetic trait $\chi$ in a similar manner to the risk sensitivity trait in Hintze et al. (2015). Here, $\chi$, is used to determine both the level of investment in a given round of the public goods game, and to guide mobility or group switch decision (see Section 3.5). Thus for each agent, $i$, it is necessary to map the value of $\chi_{i}$ to a specific investment level $x_{i}$ in each round of the public goods game. Equation 3 describes how this is done:

$x_{i}= \begin{cases}p \times V & p=1-\chi_{i} \\ 0 & p=\chi_{i}\end{cases}$

where $p$ (a uniform random variable) is the probability of investing the corresponding $x_{i}$ in the current round of the game; and $V$ is the maximum possible investment. The contribution $x_{i}$ is thus defined by a Bernoulli random variable representing the expressed behaviour or 'phenotype' of an individual given the underlying 'genotype' $\chi_{i}$. When $\chi_{i}>0.5$, there is a low chance of investing a small amount consistent with risk-averse behaviour. When $\chi_{i}<0.5$, there is a high chance of investing a large amount consistent with risk-seeking behaviour. If $\chi_{i}=p$, then the investment is zero (a form of probabilistic participation, where an agent 'free-rides').

After each individual agent $i$ has made an investment decision $x_{i}$, the accumulated investments of the $n$ group members are multiplied by a factor $k$ and evenly redistributed between the members of the group based on Equation 1. The reward (or payoff) $\pi_{i}$ is then allocated to each agent. The payoff is also added to an individual's assets (initialised as $V$ ). It is important to note that we include the accumulated assets simply as an additional metric to query/display rather than using it to guide/influence decision-making.

\subsection{Updating the risk aversion trait}

Individuals have an opportunity to update their 'strategy' or risk sensitivity trait at the end of each round of the game. Given that $\chi_{i}$ defines an individual's strategy, this equates to single trait update via social imitation. The fitness of an individual is determined relative to the performance of their current group. The more successful individuals (larger $\pi_{i}$ values) will be imitated by others, so that the number of individuals adopting a given risk trait $\chi$ will evolve over time. Thus at each time step, an individual $i$ can adopt the strategy $\chi_{j}$ from individual $j$ within its current groups using the well-known Fermi update rule (Szabó and Hauert, 2002):

$$
\chi_{i} \leftarrow \chi_{j}=\frac{1}{1+\exp \left[\left(\pi_{i}-\pi_{j}\right) / T\right]}
$$

where $T$ quantifies the uncertainty by strategy adoptions (without loss of generality we use $T=0.1$ ). All individuals are also subject to mutation. That is, with probability $\mu$ the offspring mutates to a random strategy; otherwise its risk trait $\chi_{i}$ is identical to its parent. Hence, an individual's risk sensitivity trait (or strategy) adjustment dynamics are driven by reinforcement feedback based on the individual's recent experience.

\subsection{Switching groups}

There are many different ways to implement migration models in multiple group social dilemma games. Pichugin et al. (2015) provides a detailed analysis of the available techniques.

We limit our analysis to three alternative 'group switching' strategies. Here, the overarching goal is to mediate the assortment of risk sensitivity traits from one group to another (West et al., 2007; Hintze et al., 2015). An important feature of our multiple group framework is the fact that each group is a fixed size that does not change during the simulation (this is in stark contrast to Killingback et al. (2006) but it is consistent with the approach used by Hintze et al. (2015)). Given this capacity constraint, there is no guarantee that any particular group will accept a 'switch group' request. Groups with less movement out will have less movement in. Assortment under similar capacity constraints has been shown to enhance cooperation (Smaldino and Lubell, 2011).

\subsubsection{Random migration}

In the random migration model, a fixed proportion of individuals $\lambda$ are randomly selected to migrate to a different group. Here, $\lambda$ is assumed to be very small compared to the rate of fixation of a strategy within a group (Pichugin et al., 2015) and is typically a value of 0.05 consistent with the mechanism used in islandbased evolutionary algorithms (Cantú-Paz, 1998). For each migrant, a randomly selected individual from a randomly selected group is simply nominated to 'swap' groups. This naive group-based model approximates the scenario of individuals evolving in small groups with some level of inter-group mobility. 


\subsubsection{Conditional migration}

In the conditional migration model, individuals playing the game use 'environmental' feedback as a mechanism to trigger migration. Here, individuals have the capacity to detect and leave low-quality social environments and share specific information about past group performance. We implement a form of the so-called walkaway-rule, which introduces a threshold value that defines the minimal payoff (return) an individual must receive in order to stay in the same group, otherwise the individual attempts to migrate to another group. To simplify the model, the threshold is based on the payoff $\pi$ in the given round - any profit equates to a 'good' environment.

Whether the attempted move is successful or not depends on the relative fitness of a randomly selected individual in a nominated destination group, a technique adopted in (Chiong and Kirley, 2012, 2013). Two different techniques are used to select the destination group: (a) select the 'best' group, and (b) select the 'worst' group, where we use the group payoff at the previous game iteration to determine the nominated group. The rationale behind the conditional migration models is based on the idea that a defecting individual has a higher probability of fixation in a group of cooperators as compared with a group of defectors. The fitness value of the migrant is subsequently compared with the randomly selected individual from the nominated group, and if it has a higher fitness value, the swap is complete. The aim of this assortment mechanism is to the reduce exploitation by selfish group members.

\subsubsection{Risk-sensitive migration}

A decision to migrate is made with incomplete information, where the goal is to enhance survivability and ultimately enhance fitness (and indirectly reproductive success). In the risk-sensitive migration model, we combine some of the features from the random migration model and the environmental feedback mechanism from the conditional migration model. However, what clearly differentiates this approach from the previous migration models is the use of the risk sensitivity trait, $\chi$, in the decision making process. Here, $\chi$ endogenously determines an individual's propensity to switch groups, if a 'satisfaction' threshold is not met. Importantly, this risk-sensitive model is a form of 'blind switching' where individuals attempting to swap groups, do so without specific knowledge of the payoff or social rewards of other groups. All individuals who have flagged their intention to migrate are moved to an external 'migrant pool.' Then each of the individuals from this 'migrant pool' are randomly allocated back to one of the groups. This approach allows for heterogeneity in risk preferences. Importantly, risk-seeking individuals tend to seek out a new, potentially more profitable, group.

\section{Simulation experiments}

A series of Monte Carlo simulation experiments were performed to examine population dynamics in our model. Two questions guided the experimental design:

1. Is it possible to evolve risk-seeking behaviour in the multiple group public goods game?

2. Given capacity constraints for group size, does risksensitive conditional migration offer greater opportunities for positive assortment when compared to random migration and other forms of conditional migration?

In all simulation experiments, the value of $\chi$ was initialised randomly from a uniformly distribution at the beginning of a trial. The value of the maximum investment $V$ and the $M P C R$ were 5.0 and 0.75 respectively. In the reproduction stage, the mutation rate $\mu$ was 0.01 . The migration rate $\lambda$ was set to 0.05 .

In the conditional migration model, the threshold value used to determine whether an agent attempts to switch groups was simply the payoff value in the current round - a loss corresponding to a low-quality social environments. The payoff performance from each group was made available to all individuals. In the risksensitive migration model, each 'switch group' request was based on the risk sensitivity trait $\chi$. However, the payoff performance from other groups was not available.

All results listed below are mean values over 30 simulation trials with differing seeds for the random number generators. Error bars have been omitted from most time-series plots as they are smoothed values and the standard errors are very small. Key model parameters investigated are: group size $(n)$, the number of groups $(g)$, and the group switching (or migration) mechanism (random, conditional - best or worse, risk-sensitive). Key output metrics include: evolved $\chi$ values - both within and between groups and assortment levels, measured in terms of the variance ratio for $\chi$ both between and across groups. We also report limited results for cumulated assets (profit) to further illustrate the efficacy of the model.

\subsection{Evolutionary trajectory of $\chi$ in a single group}

In the first set of experiments, we investigate the evolutionary trajectory of $\chi$ in a single group $(g=1)$ with 


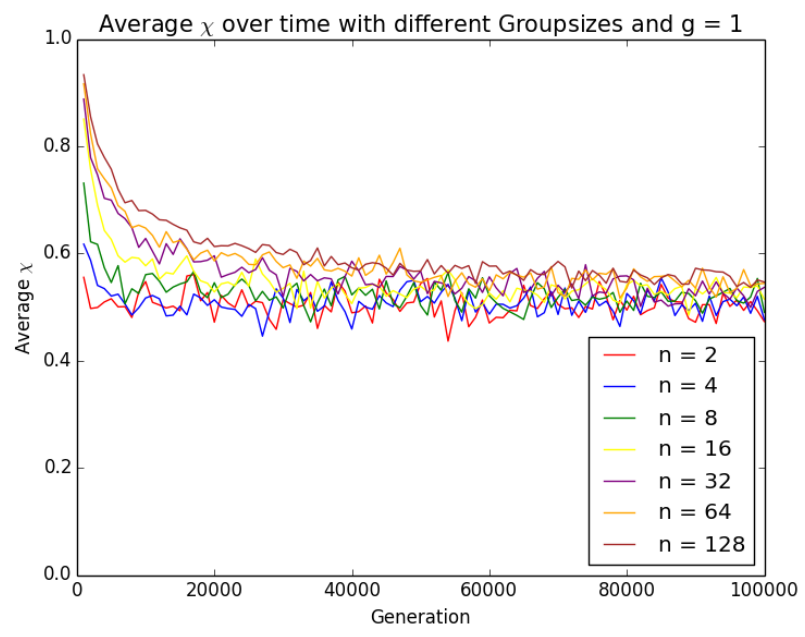

Fig. 2: The average strategy $\chi$ vs generation number across the population for varying population size $n$ when $g=1$.

varying population sizes $n \in\{2,4,8,16,32,64,128\}$ and constant $M P C R$ value. The simulations start from a random uniform initial distribution for $\chi$.

Fig. 2 plots time series values for the average strategy $\chi$ vs generation number for different $n$ values. For all values of $n$, the trajectory of $\chi$ fluctuates around the mid-range value of 0.5 , after initial evolutionary selection pressure favoured larger values of $\chi$ at earlier time steps. Significantly, the number of steps required to reach this value is correlated with the population size, a result consistent in general with the risk sensitivity model of Hintze et al. (2015). This suggests that the size of the group must be considered when analysing perceptions of risk in the continuous public goods game. For smaller population sizes, there appears to an emergent preference for $\chi$ with low variance (see sub-section 4.3 for further details).

\subsection{Evolutionary trajectory of $\chi$ in multiple groups}

In the second set of simulation experiments, we investigate the underlying population dynamics, limiting the analysis to two migration models - random migration and conditional migration. That is, mobility decisions are not based directly on the 'genetic trait' $\chi$. Fig. 3 plots the average value of $\chi$, calculated over the final 2500 generations of a simulation trial, for each of the different group sizes considered $n \in\{2,4,8,16,32,64,128\})$ with $g=32$ groups. Errors bars have been included on the plot. Based on Wilcoxon rank sum test, there are significant differences between each of the migration modes consider $(p<0.01)$ for

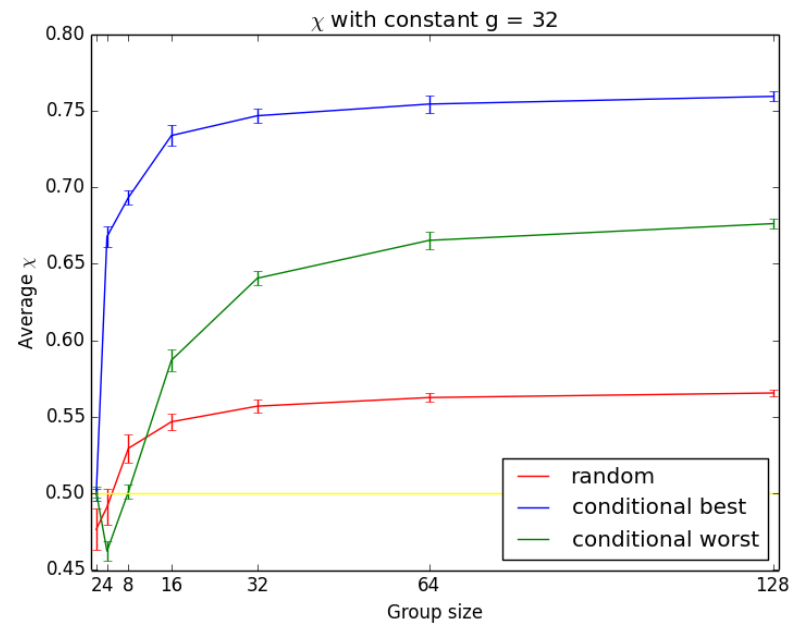

Fig. 3: The average strategy $\chi$ across the population for varying groups sizes calculated over the last 2500 generations for each of the migration model. The yellow line indicates the expected value of 0.5 for unbiased evolution; i.e. no risk bias/preference.

larger group sizes. When the group size is smaller $(n=2$ and $n=4$ ) the differences are not as clear-cut. This is to be expected as the game is relatively easy (event though there is still a social dilemma) under this condition. It is important to note that as the size of the group increases there is transition to higher average $\chi$ values.

Fig. 4 plots trends in the trajectory of the risk sensitivity trait $\chi$. When $n=4$, the differences between the random migration and conditional worst migration strategy are not significant, with the $\chi$ fluctuating around the 0.5 value. However, in the case of conditional best migration, the $\chi$ fluctuates around a value of 0.7 , indicating relative high levels of average risk aversion across the population. However, when the group size is larger $(n=32)$, the evolutionary trajectory of the $\chi$ changes considerably. In each of the migration models considered, the average value of $\chi$ is greater than the expected unbiased value of 0.5 . The selection pressured embedded in the conditional migrations results in higher $\chi$ values, with average values around 0.8 emerging in the conditional best model.

A closer inspection of the number of a successful 'group switches' is necessary, give the constraints imposed by the fixed group size. Fig. 5 plots time series values for the proportion of migrant 'desired' and 'successful' moves respectively for a typical population size ( $g=32$ and $n=32$ in this case). Here, 'desired' simply means that an individual has flagged their intention that they wish to swap groups as the payoff received 


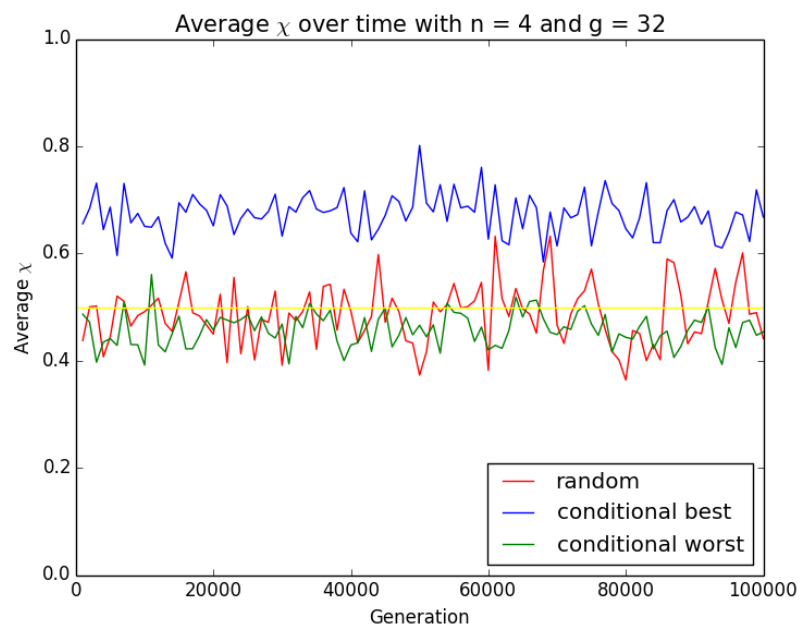

(a) $n=4$

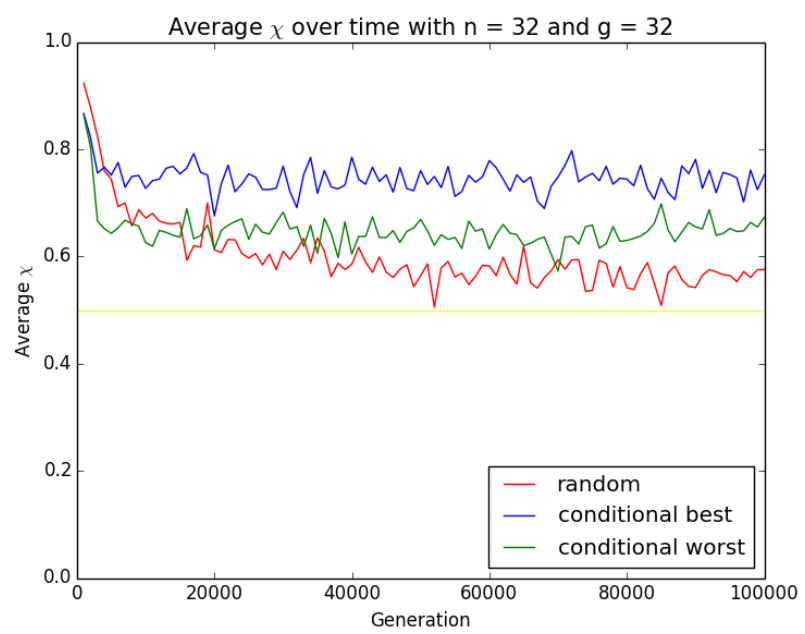

(b) $n=32$

Fig. 4: Average strategy $\chi$ vs generation number for each of the migration models considered. The expected value of 0.5 for unbiased evolution is also plotted.

in the current game was not satisfactory. A successful move is when the individual actually switches groups. As expected, the proportion of successful moves is significantly lower than desired moves in the conditional modes, with lowest values apparent in the conditional best migration mode. This result is consistent with the expectation that potential risky moves may have a positive effect on assortment.

To further assess population dynamics, we plot time series values of accumulate average profit (based on accumulated payoff values, $\pi$ ) for agents in Fig. 6. Clear differences can been seen between migration modes in both plots. A comparison between the two different

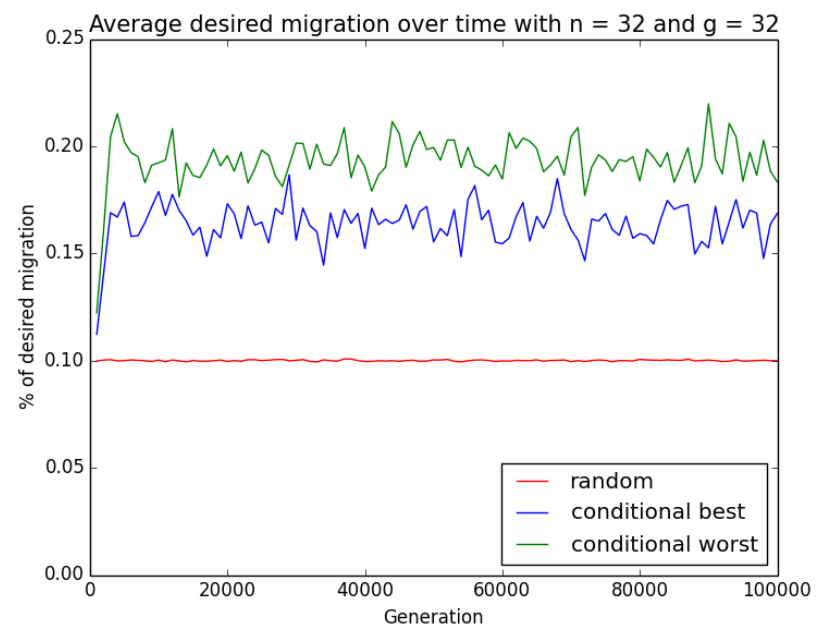

(a) Desired

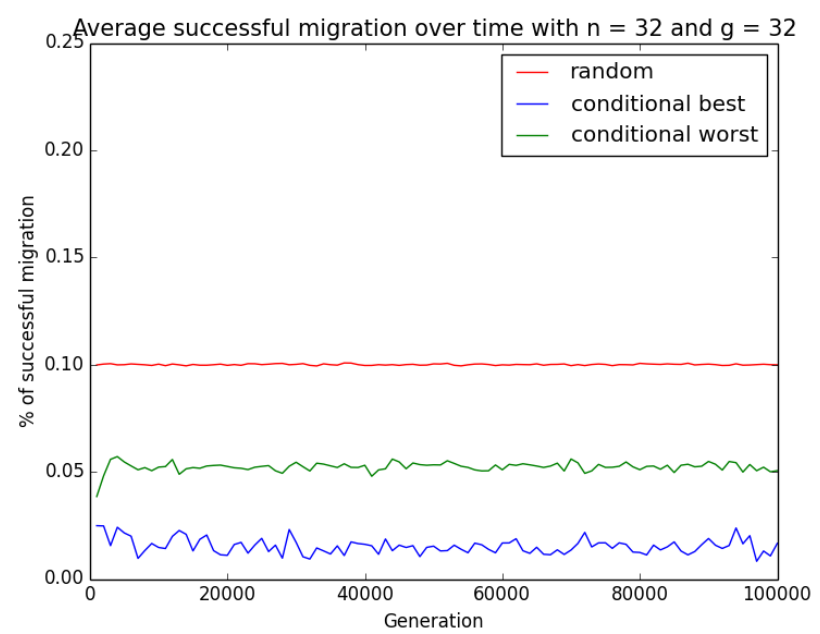

(b) Successful

Fig. 5: Proportion of (a) desired and (b) successful moves vs generation number for each of the migration models when group size $n=32$ and $g=32$.

group sizes ( $n=4$ and $n=32$ ) reveals that for larger groups the average profit decreases. The differences between the conditional best and conditional worse modes are apparent in both group sizes. The results suggest that this transition to risk averse behaviour, especially in larger groups, is caused by the presence of 'freeriders.' Since the investment decision is based on both the risk sensitivity trait and the expectation of other group members, we observe a qualitatively different relationship between risk-averse investment levels and group size. Conditional migration provides opportunities to take advantage of potentially more beneficial 'social environments,' while allowing for the possibility 


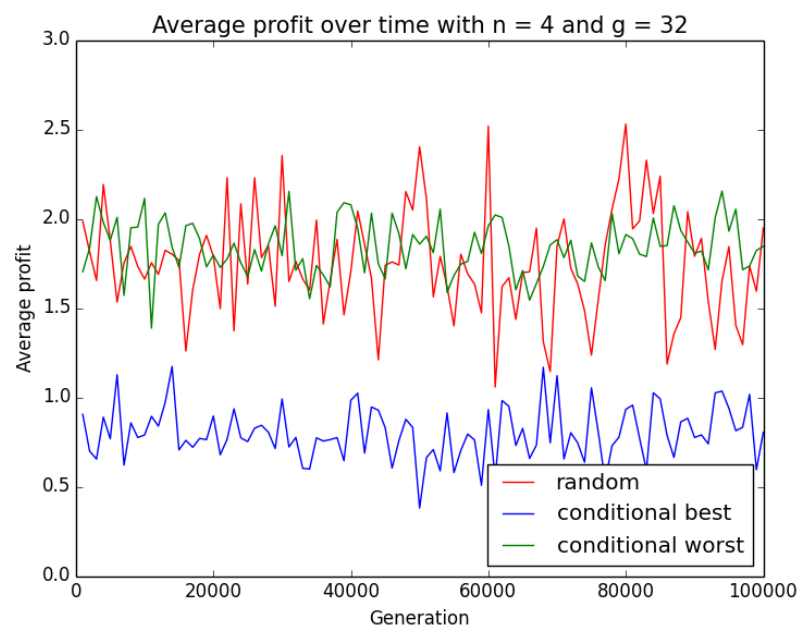

(a) $n=4$

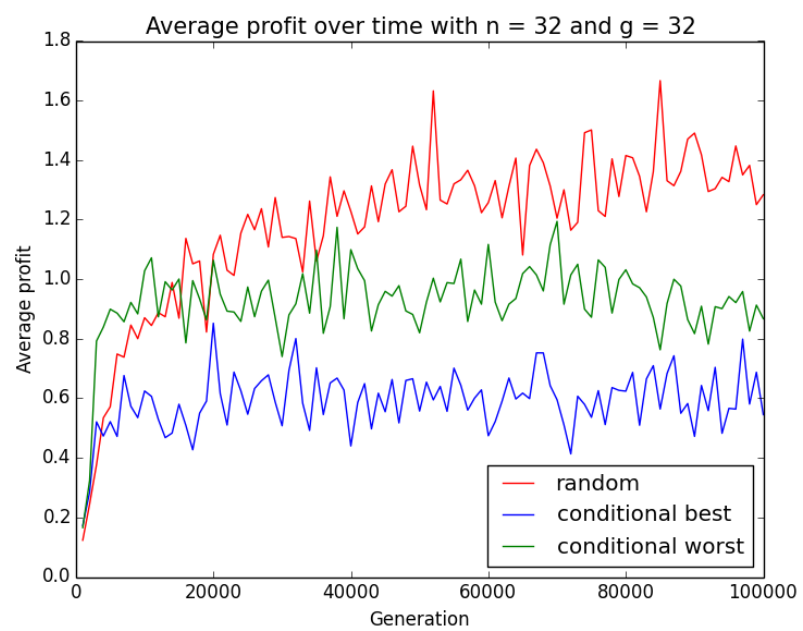

(b) $n=32$

Fig. 6: Average profit vs generation number for each of the migration models.

of leaving a group when that particular environment degrades. A constraint on conditional migration was that the migrant must have higher fitness value than the randomly selected individual from the nominated group (either the best or worse group depending on the model). This constraint was imposed to mediate the assortment effects. However, such an approach requires that an individual can query the 'performance' of other groups in the population.

\subsection{Risk-sensitive assortment}

The previous simulation experiments have provided important insights into the population dynamics of the multiple group public goods game. The results suggest that decision-making based on a level of risk sensitivity leads to interesting outcomes. The focus of our analysis now turns to understanding assortment within the model in more detail. We are particular interested in examining the effects that assortment has on the evolutionary trajectory of both individual $\chi$ values and the population wide distribution of $\chi$ values, when mobility (or group switch) requests are based on threshold satisfaction levels and the 'genetic trait' $\chi$.

Additional simulation experiments were carried out to investigate the group size effects on the level of investment in the public goods game by keeping the $M P C R$ ratio fixed ${ }^{1}$ when increasing the size of the group. Fig. 7 plots the average 'genetic trait' $\chi$ across the population at the end of the simulation for varying group size $(n)$ and number of group $(g)$ when $M P C R=0.75$. As expected, the overall trend is consistent with the model of Hintze et al. (2015), with values relatively close to the un-biased value of 0.5 . However, an inspection of the plot suggests that risksensitive assortment has emerged, especially for larger group sizes. For a given $\chi$ value (or range of genotype values) to increase in frequency in a population, individuals must, on average, end up with higher fitness benefits (payoff values) than average population members. That is, the local group imitation dynamics coupled with risk-sensitive migration must be guiding the trajectory of the evolving population. Individuals who attempt to switch groups are searching for a more cooperative group. However, further analysis of assortment and genetic variation is required before firm conclusions can be made.

To delve deeper, a version of a 'relatedness' metric for single trait evolving populations is used to measure assortment in the multiple group public goods game. This metric is based on Hamilton's rule (Hamilton, 1964). Similar versions have been used by Fletcher and Zwick (2007) and Pepper and Smuts (2002). Here, the 'assortment ratio' $r$ is defined as:

$r=\frac{V_{B}}{V_{B}+\overline{V_{W}}}$

where $V_{B}$ and $\overline{V_{W}}$ are the statistical variance of the $\chi$ values between groups and the average statistical variance of the $\chi$ values within groups, respectively. Thus, $r$ can be used as a similarity or assortment metric, representing the between-group variance over the total variance.

1 We have also run a large number of simulations using a range of $V$ and $k$ values. The results were qualitatively the same across a range of values, thus we do not include all results in this paper. 

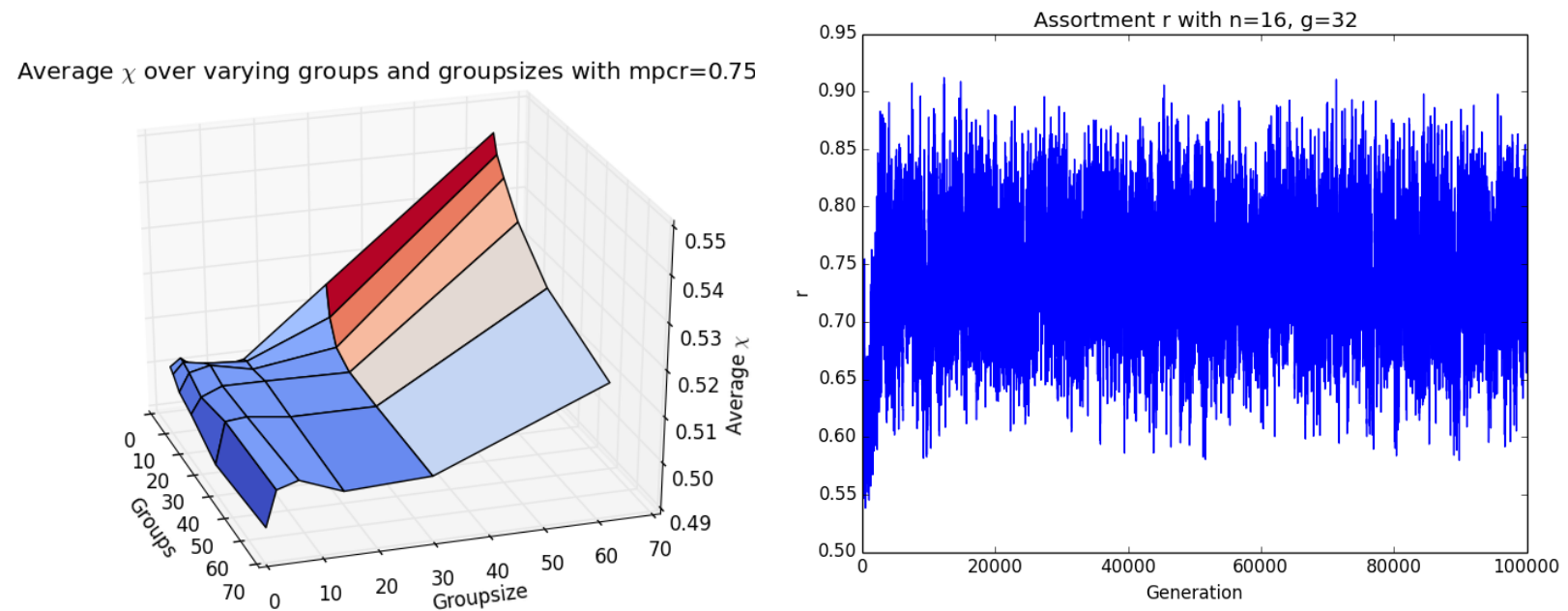

Fig. 7: The average strategy $\chi$ across the population for varying group size $(n)$ and number of groups $(g)$ calculated over the last 2500 generations when $M P C R=$ 0.75 .

Average $r$ over varying groups and groupsizes with $\mathrm{mpcr}=0.75$

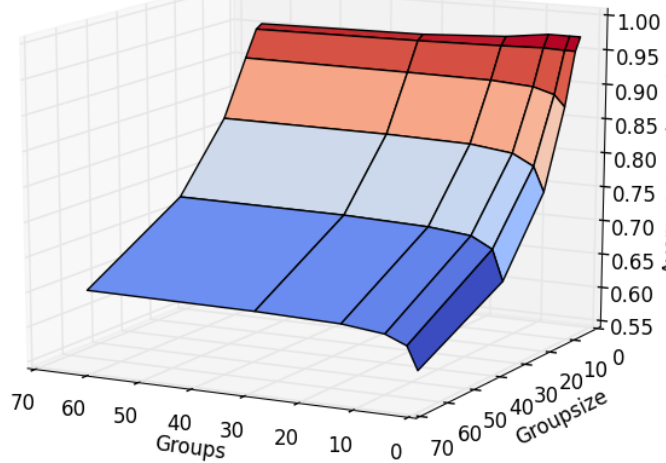

1.00
-0.95 95 $0.90 \stackrel{0}{\mathrm{~d}}$ 0.75 cantly lower, suggesting the presence of 'free riders,' - 0.70 융 distributed throughout the groups.

Fig. 8: The assortment ratio $r$ value averaged over the last 2500 generations for varying group size $(n)$ and number of groups $(g)$ when $M P R C=0.75$.

Fig. 8 plots the relationship between group size, the number of groups and the emergent assortment levels (in terms of the ratio of the variance of the $\chi$ values within the model). For smaller groups, the value of $r$ is closer to 1 , suggesting that ratio of the variation (or diversity) of $\chi$ values in each group, and between groups, is relatively low. When continuous investment is used
To conclude this section, we plot time series values of the assortment level $r$ for one scenario $(n=16$ and $n=32)$ in Fig. 9. Despite the overall trends suggested in Figs. 7 and 8, there are significant fluctuations in the trajectory of assortment ratio values at each time step reflecting the game dynamics.

Fig. 9: The assortment ratio $r$ vs generation number when $g=32$ and $n=16$ when $M P C R=0.75$.

in the multiple group public goods game, it is possible for individual groups to be homogenous, however, more than likely the groups will contain a mix of individuals making small contributions or be a mix of a small number of 'cooperators' making substantial consults shown in the plot are consistent with observations reported in Gavrilets (2015), where increasing group ize typically decreases within-group cooperation. The trends in Fig. 8 indicate high emergent altruism lev-

\section{Discussion and conclusions}

In this paper we have described a hybridised version of a multiple group public goods game / risk sensitive model. By explicitly adopting an evolutionary game theoretic approach, where risk-seeking can be conceived as potentially beneficial, we have examined the most basic requirement for the evolution of 'cooperative behaviour' based on positive assortment. The rationale behind our model was based on the fact that uncertainty and risk are key features of many social dilemmas, where individual decisions are often made with imperfect knowledge and variance in outcomes. Individual tributions and a large number of 'defectors.' The re- 
game playing agents adopted a continuous investment strategy mapped to their underlying risk sensitivity parameter. Additionally, we allowed this risk sensitivity trait to be used in the 'group switching' decision. Thus, a dynamic perception of risk was used to guide both investment levels and mobility.

In multiple group games with variable group sizes, the general outcome of the game is correlated with both the average group size and the variance of the groupsize distribution (Killingback et al., 2006; Pena, 2012). However, given the constraints of fixed group sizes in our model, it is reasonable to expect that emergent dynamics may be different (Szolnoki and Perc, 2011). The simulation results listed in Section 4 illustrate that the mean level of the risk aversion trait $\chi$ increases over time, especially for larger groups, a result consistent with Gavrilets (2015), who report that larger group size typically makes it more difficult for cooperation to be maintained in cooperative dilemma games. In our model, lower investment levels corresponding to risk-averse behaviour were evident, a result consistent with the work of (Sella and Lachmann, 2000) in social dilemma games and the work of Hintze et al. (2015) focussed on the adaptive benefits of risk-aversion in an evolutionary context.

An analysis of the alternative mobility or migration models reveals interesting insights. The random migration model introduces a limited level of assortment. In contrast, conditional migration significantly affects population assortment. In the 'blind switch' risksensitive migration model, the emergent value of $r$ is inversely correlated with the groups size. The average value of $\chi$ across the population at the end of the simulation run was also correlated with both the group size, and to a lesser extent the number of groups. Here, mobility can be thought of as a secondary goal, in that requests to switch groups are made on the basis that a successful move will lead to higher rewards (the primary goal). Population viscosity is a self-organising process that depends only on the risk each individual is exposed to, and is willing to accept. When an individual can 'query' other groups before attempting to migrate to a new group, the dynamics change. In the conditional worse mode, the proportion of successful switches is greater as, on average, the fitness of individuals in the worse pool will be relatively small, thus the chance of a successful move increases. However, the group may contain many free-riders, which adversely affect future performance. In the conditional best mode, the chance of a successful switch are reduced, but if successful, the positive assortment leads to higher future return. In each of the conditional migration models, capacity constraints impose a fixed upper limit on the number of individuals that can join any particular groups, which directly impacts on the evolutionary trajectory of the risk aversion trait.

In sum, detailed simulation experiments confirm a number of our expectations about the relationship between risk orientation, decision-making and mobility in the multiple-group public goods game. As the size of the group increases, in the long term, assortment levels tend to decrease and risk-averse individuals tend to dominate the population. This suggests that the ability to respond contingently to the social environment via a risk sensitivity trait - is a desirable characteristic to have. Significantly, a positive correlation between evolutionarily stable levels of altruism and mobility do emerge in the multiple group public goods game.

There are a number of directions to explore in future work. In our model, we did not impose additional costs when changing groups. Exploring the effect of such costs and limiting the number of possible moves is an interesting direction for future empirical research. It would also be interesting to examine more complex rules for migration/mobility including social networks and geographical locations as discussed in Pfau et al. (2013). Despite the robustness of our findings, further application in real social psychology settings may be problematic. If individuals are regularly faced with fluctuations of their general environment - variable risky situations - the proposed model should be tested experimentally to validate its conclusions.

\section{References}

Melissa Bateson. Recent advances in our understanding of risk-sensitive foraging preferences. Proceedings of the Nutrition Society, 61(04):509-516, 2002.

Alison M Bell. Approaching the genomics of risk-taking behavior. Advances in Genetics, 68:83-104, 2009.

Robert Boyd and Peter J Richerson. Group beneficial norms can spread rapidly in a structured population. Journal of theoretical biology, 215(3):287-296, 2002.

E. Cantú-Paz. A survey of parallel genetic algorithms. Calculateurs Parallèles, Réseaux et Systòmes Répartis, 10(2):141-171, 1998.

Valerio Capraro. A model of human cooperation in social dilemmas. PLoS One, 8(8):e72427, 2013.

Valerio Capraro, Jillian J Jordan, and David G Rand. Heuristics guide the implementation of social preferences in one-shot prisoner's dilemma experiments. Scientific reports, 4, 2014.

Raymond Chiong and Michael Kirley. Random mobility and the evolution of cooperation in spatial $N$-player iterated prisoner's dilemma games. Physica A: Statis- 
tical Mechanics and its Applications, 391:3915-3923, 2012.

Raymond Chiong and Michael Kirley. A multi-agent based migration model for evolving cooperation in the spatial n-player snowdrift game. In PRIMA 2013: Principles and Practice of Multi-Agent Systems, pages 70-84. Springer, 2013.

Ross Cressman, Jie-Wen Song, Bo-Yu Zhang, and Yi Tao. Cooperation and evolutionary dynamics in the public goods game with institutional incentives. Journal of Theoretical Biology, 299:144-151, 2012.

Michael Doebeli, Ulf Dieckmann, and Leen Hordijk. Adaptive dynamics of speciation: spatial structure. Adaptive speciation, pages 140-167, 2004.

Jeffrey A Fletcher and Martin Zwick. The evolution of altruism: Game theory in multilevel selection and inclusive fitness. Journal of Theoretical Biology, 245 (1):26-36, 2007.

F. Fu and M.A. Nowak. Global migration can lead to stronger spatial selection than local migration. J Stat Phys, (3-4):637-653, 2013.

Feng Fu, Xiaojie Chen, Lianghuan Liu, and Long Wang. Social dilemmas in an online social network: the structure and evolution of cooperation. Physics Letters $A, 371(1-2): 58-64,2007$.

Sergey Gavrilets. Collective action problem in heterogeneous groups. Phil. Trans. R. Soc. B, 370(1683): 20150016, 2015.

Herbert Gintis. Game theory evolving: A problemcentered introduction to modeling strategic behavior. Princeton University Press, 2000.

William D Hamilton. The genetical evolution of social behaviour. ii. Journal of Theoretical Biology, 7(1): 17-52, 1964.

Garrett Hardin. The tragedy of the commons. science, 162(3859):1243-1248, 1968.

Christoph Hauert. Cooperation, Collectives Formation and Specialization. Advances in Complex Systems, 09(04):315-335, December 2006. ISSN 0219-5259.

Christoph Hauert, Yu-Ting Chen, and Lorens A Imhof. Fixation times in deme structured, finite populations with rare migration. Journal of Statistical Physics, 156(4):739-759, 2014.

D. Helbing and W. Yu. The outbreak of cooperation among success-driven individuals under noisy conditions. Proceedings of the National Academy of Sciences of the United States of America, 106:36803685, 2009.

Arend Hintze, Randal S Olson, Christoph Adami, and Ralph Hertwig. Risk sensitivity as an evolutionary adaptation. Scientific reports, 5, 2015.

Marco Janssen and Robert Goldstone. Dynamicpersistence of cooperation in public good games when group size is dynamic. Journal of Theoretical Biology, 243(1):134-42, November 2006.

L.-L. Jiang, W.-X. Wang, Y.-C. Lai, and B.-H. Wang. Role of adaptive migration in promoting cooperation in spatial games. Physical Review E, 81(3):036108, 2010.

Daniel Kahneman and Amos Tversky. Prospect theory: An analysis of decision under risk. Econometrica: Journal of the Econometric Society, pages 263-291, 1979.

Kiridaran Kanagaretnam, Stuart Mestelman, Khalid Nainar, and Mohamed Shehata. The impact of social value orientation and risk attitudes on trust and reciprocity. Journal of Economic Psychology, 30(3): 368-380, 2009.

Timothy Killingback, Jonas Bieri, and Thomas Flatt. Evolution in group-structured populations can resolve the tragedy of the commons. Proceedings of the Royal Society of London B: Biological Sciences, 273(1593):1477-1481, 2006.

Michael Kirley and Friedrich Burkhard von der Osten. Adaptive risk aversion in social dilemmas. In Simulated Evolution and Learning, pages 443-454. Springer, 2014.

Jean-François Le Galliard, Régis Ferriere, and Ulf Dieckmann. Adaptive evolution of social traits: origin, trajectories, and correlations of altruism and mobility. The American Naturalist, 165(2):206-224, 2005.

Michael W Macy. Pavlov and the evolution of cooperation: An experimental test. Social Psychology Quarterly, pages 74-87, 1995.

Martin Nowak. Five rules for the evolution of cooperation. Science, 314(5805):1560-3, December 2006.

Martin Nowak. Evolving cooperation. Journal of Theoretical Biology, 299:1-8, April 2012. ISSN 1095-8541.

Mancur Olson. The logic of collective action: Public goods and the theory of groups, second printing with new preface and appendix (harvard economic studies). 1971

Craig D Parks. Risk preference as a predictor of cooperation in a social dilemma. Contemporary psychological research on social dilemmas, pages 58-70, 2004.

Jorge Pena. Group-size diversity in public goods games. Evolution, 66(3):623-636, 2012.

John W Pepper and Barbara B Smuts. A mechanism for the evolution of altruism among nonkin: positive assortment through environmental feedback. The American Naturalist, 160(2):205-213, 2002.

Matjaž Perc, Jesús Gómez-Gardeñes, Attila Szolnoki, Luis M Floría, and Yamir Moreno. Evolutionary dynamics of group interactions on structured popula- 
tions: a review. Journal of The Royal Society Interface, 10(80), 2013.

Jens Pfau, Michael Kirley, and Yoshihisa Kashima. The co-evolution of cultures, social network communities, and agent locations in an extension of axelrod's model of cultural dissemination. Physica A: Statistical Mechanics and its Applications, 392(2):381-391, 2013.

Yuriy Pichugin, Chaitanya S Gokhale, Julián Garcia, Arne Traulsen, and Paul B Rainey. Modes of migration and multilevel selection in evolutionary multiplayer games. Journal of Theoretical Biology, 387: 144-153, 2015.

Tatsuya Sasaki, Isamu Okada, and Tatsuo Unemi. Probabilistic participation in public goods games. Proceedings of the Royal Society B: Biological Sciences, 274(1625):2639-2642, 2007.

Guy Sella and Michael Lachmann. On the dynamic persistence of cooperation: How lower individual fitness induces higher survivability. Journal of Theoretical Biology, 206(4):465-485, 2000.

Daniel B Shank, Yoshihisa Kashima, Saam Saber, Thomas Gale, and Michael Kirley. Dilemma of dilemmas: How collective and individual perspectives can clarify the size dilemma in voluntary linear public goods dilemmas. PloS One, 10(3), 2015.

Paul E Smaldino and Mark Lubell. An institutional mechanism for assortment in an ecology of games. PLoS One, 6(8):e23019, 2011.

Michael D Stern. Patrimony and the evolution of risktaking. PloS One, 5(7):e11656, 2010.

György Szabó and Christoph Hauert. Phase transitions and volunteering in spatial public goods games. Physical Review Letters, 89(11):118101, 2002.

Attila Szolnoki. Promoting cooperation in social dilemmas via simple coevolutionary rules. The European Physical Journal B-Condensed Matter and Complex Systems, 67(3):337--344, 2009.

Attila Szolnoki and Matjaž Perc. Group-size effects on the evolution of cooperation in the spatial public goods game. Physical Review E, 84(4):047102, 2011.

Arne Traulsen and Martin A Nowak. Evolution of cooperation by multilevel selection. Proceedings of the National Academy of Sciences, 103(29):10952-10955, 2006.

Mendeli H Vainstein, Ana TC Silva, and Jeferson J Arenzon. Does mobility decrease cooperation? Journal of Theoretical Biology, 244(4):722-728, 2007.

M Van Assen and Chris Snijders. Effects of risk preferences in social dilemmas: a game-theoretical analysis and evidence from two experiments. Contemporary psychological research on social dilemmas., pages 38 65,2004 .
Stuart A West, Ashleigh S Griffin, and Andy Gardner. Social semantics: altruism, cooperation, mutualism, strong reciprocity and group selection. Journal of Evolutionary Biology, 20(2):415-432, 2007.

David Sloan Wilson and Lee A Dugatkin. Group selection and assortative interactions. American Naturalist, pages 336-351, 1997.

H.-X. Yang, Z.-X. Wu, and B.-H. Wang. Role of aspiration-induced migration in cooperation. Physical Review E, 81(6):065101, 2010.

Weijun Zeng, Minqiang Li, Fuzan Chen, and Guofang Nan. Risk consideration and cooperation in the iterated prisoner's dilemma. Soft Computing, pages 1-21, 2014. 


\section{University Library}

\section{- M M I N E R VA A gateway to Melbourne's research publications}

Minerva Access is the Institutional Repository of The University of Melbourne

Author/s:

Kirley, M;von der Osten, FB

Title:

Risk sensitivity and assortment in social dilemmas

Date:

2016-10-01

Citation:

Kirley, M. \& von der Osten, F. B. (2016). Risk sensitivity and assortment in social dilemmas. SOFT COMPUTING, 20 (10), pp.3775-3786. https://doi.org/10.1007/s00500-016-2090-5.

Persistent Link:

http://hdl.handle.net/11343/282739 